\title{
Autonomia e gerenciamento do enfermeiro no serviço de cirurgia robótica
}

\section{RESUMO}

Objetivo: Caracterizar a prática do gerenciamento do Enfermeiro em Cirurgia Robótica. Método: Trata-se de uma Revisão Integrativa da Literatura (RIL) de artigos publicados em português e inglês, disponiveis na íntegra nas bases da dados da LILACS e PubMed, tendo como recorte temporal os anos de 2008 a 2019. Utilizados os descritores "Enfermagem", "Robótica" e "Centro Cirúrgico", sendo selecionados e analisados oito estudos. Resultados: Os anos de maiores publicações foram 2016, com n= 02 estudos e $2017 \mathrm{com} \mathrm{n=} 02$ estudos. A base de dados com maiores resultados foi a PubMed com $n=05$. Evidencia que as principais publicações sobre cirurgias robóticas concentram-se na área médica com enfoque na técnica cirúrgica, apontando uma carência de estudos com o foco na enfermagem. Conclusão: 0 enfermeiro atuante em cirurgia robótica deve ser dinâmico, estar constantemente atualizando seus conhecimentos com o intuito de treinar e gerenciar sua equipe em todo o processo que ocorre na sala cirúrgica.

DESCRITORES: Enfermagem; Robótica; Centro Cirúrgico.

\section{ABSTRACT}

Objective: Characterize the practice of nurse management in robotic surgery. Method: This is an Integrative Literature Review (RIL) of articles published in Portuguese and English, available in full in the LILACS and PubMed databases. "', Robotics "and" Surgical Center ", being selected and analyzed eight studies. Results: The years of largest publications were 2016, with $n=02$ studies and 2017 with $n=02$ studies. The database with the highest results was PubMed with $n=05$. It shows that the main publications on robotic surgery are concentrated in the medical area, focusing on the surgical technique, indicating a lack of studies focusing on nursing. Conclusion: The nurse working in robotic surgery must be dynamic, constantly updating their knowledge in order to train and manage their team throughout the process that occurs in the operating room.

KEYWORDS: Nursing; Robotics; Surgical Center.

\section{RESUMEN}

Objetivo: caracterizar la práctica del manejo de enfermeras en cirugía robótica. Método: Esta es una Revisión Integral de Literatura (RIL) de artículos publicados en portugués e inglés, disponible en su totalidad en las bases de datos LILACS y PubMed, con el marco temporal de 2008 a 2019. Utilizando las palabras clave "Enfermería". "', Robótica "y" Centro quirúrgico ", siendo seleccionados y analizados ocho estudios. Resultados: Los años de las principales publicaciones fueron 2016, con $n=02$ estudios y $2017 \operatorname{con} n=$ 02 estudios. La base de datos con los resultados más altos fue PubMed con $n=05$. Muestra que las principales publicaciones sobre cirugía robótica se concentran en el área médica, centrándose en la técnica quirúrgica, lo que indica una falta de estudios centrados en enfermería. Conclusión: La enfermera que trabaja en cirugía robótica debe ser dinámica, actualizando constantemente sus conocimientos para capacitar y administrar a su equipo durante todo el proceso que ocurre en la sala de operaciones.

PALABRAS CLAVE: Enfermería; Robótica; Centro Cirúrgico.

RECEBIDO EM: 19/11/2019 APROVADO EM: 22/11/2019

\section{Fernanda Ferreira e Silva}

Acadêmica do $10^{\circ}$ período do Curso de Graduação em Enfermagem no Centro Universitário Augusto Motta/UNISUAM-Rio de Janeiro. RJ.

\section{Priscila Ferreira dos Santos}

Acadêmica do $10^{\circ}$ período do Curso de Graduação em Enfermagem no Centro Universitário Augusto Motta/UNISUAM-Rio de Janeiro. RJ

Ana Paula Pinheiro Dalto

Acadêmica do 10 o período do Curso de Graduação em Enfermagem no Centro Universitário Augusto Motta/UNISUAM-Rio de Janeiro.RJ 


\section{artigo}

Silva, F.F.; Santos, P.F.; Dalto, A.P.P.; Granandeiro, D.S.; Granandeiro, R.M.A.; Melo, N.G.S.; Passos, J.P.

Autonomia e gerenciamento do enfermeiro no serviço de cirurgia robótica

\section{Daniel da Silva Granandeiro}

Enfermeiro. Mestre em Enfermagem pelo PPGEnf/UERJ. Doutorando em Enfermagem e Biociências pelo PPGEnfBio/UNIRIO. Bolsista DS/CAPES. Professor no Centro Universitário Augusto Motta/UNISUAM. Rio de Janeiro, RJ.

\section{Raquel Magalhães de Azeredo Granadeiro}

Enfermeira. Mestre em Enfermagem pelo PPGEnf/MPEA/UFF. Professora no Centro Universitário Augusto Motta/UNISUAM; Rio de Janeiro, RJ.

\section{Noemi Garcia Silva de Melo}

Enfermeira. Especialização em Terapia Intensiva pela UERJ. Especialização em Cardiologia pela Universidade Gama Filho. Professora no Centro Universitário Augusto Motta/UNISUAM Rio de Janeiro, RJ

\section{Joanir Pereira Passos}

Enfermeira. Doutora em Enfermagem pela Universidade de São Paulo. Professora titular da Universidade Federal do Estado do Rio de Janeiro/UNIRIO. Líder do laboratório de pesquisa: Enfermagem, tecnologias, saúde e trabalho. Rio de Janeiro, RJ.

\section{INTRODUÇÃO}

A Cirurgia Robótica surgiu no EUA pelo ano de 2000 e chegou no Brasil em 2008. Conforme dados de 2018 da própria empresa, no Brasil o Sistema Robótico está presente em 09 Estados com 41 Robôs em funcionamento, acredita-se que no ano de 2019 este número tenha aumentado. As Forças Armadas dos Estados Unidos junto com a NASA e a Darpa (Defense Advanced Research Projects Agency) tentaram desenvolver um sistema de cirurgia para campos de guerra, sem sucesso, a empresa Intuitive Surgical criou o Sistema Da Vinci Robótica.

A Cirurgia Robótica caracteriza-se como uma grande conquista da cirurgia minimamente invasiva, elevando a videocirurgia e agregando importantes avanços tecnológicos a sala de operações ${ }^{(1)}$.

$\mathrm{Na}$ Cirurgia Robótica, os instrumentos, ou pinças são mais longos e inseridos por trocarter, por onde o cirurgião trabalha na cavidade abdominal ou torácica. A diferença de uma cirurgia laparoscópica é que na cirurgia robótica o cirurgião manipula as pinças através de um Console, interligadas por um cabo. O Sistema Da Vinci de Robótica é composto por três componentes: Console do cirurgião: local de controle das ações do Robô, que possui controladores de mão e pedais, por meio dos quais o médico executa o procedimento; Carro do paciente: componente operatório formado pelos braços robóticos, local de colocação do instrumental cirúrgico (pinças robóticas) e da câmera; Carro de visão: carro que aloja o monitor de visão, o sistema de câmera e a fibra ótica, bem como as prateleiras para colocação do bisturi e de insufladores ${ }^{(1)}$.

Os serviços de Cirurgia Robótica, quando são inaugurados, prezam pela alta qualidade da equipe de enfermagem juntamente com a presença do Enfermeiro em sala. Assim, com o decorrer do tempo, vão abrindo mão dos detalhes e diminuindo cada vez mais a presença do Enfermeiro em função dos compromissos e burocracias. Dessa forma, o profissional continua sendo responsável por este serviço, mas está presencialmente fora da sala cirúrgica, envolvido em outros processos.

Com isso, o enfermeiro tem papel fundamental dentro da sala de cirurgia robótica e a prioridade é pelo planejamento do sistema robótico e pela provisão dos insumos e dos equipamentos necessários à especialidade que será operada, aos processos de proteção e à segurança do paciente, sendo este, um diferencial da cirurgia robótica. Sendo assim, levando em consideração a segurança do paciente, a presença do enfermeiro passou a ser característica fundamental e determinante para garanti-la, tendo como focos de atribuição o posicionamento do cliente e treinamento conforme curva de aprendizado de cada colaborador para manutenção de uma equipe coesa ${ }^{(4)}$.

Objetivo do estudo é caracterizar a prática do gerenciamento do enfermeiro em cirurgia robótica, tendo como questão norteadora: Qual o papel gerencial do enfermeiro na cirurgia robótica?

\section{METODOLOGIA}

Trata-se de Revisão Integrativa da Literatura (RIL) que é definida como método que reúne, avalia e sintetiza os resultados de pesquisas sobre a temática específica, no qual se percorre as seguintes etapas: elaboração da questão de pesquisa, busca na literatura dos estudos, extração de dados, avaliação dos estudos incluídos, interpretação dos resultados e apresentação da revisão.

No primeiro momento, foi definida pelos autores a questão norteadora do estudo: Qual o papel gerencial do enfermeiro na cirurgia robótica?

A coleta do material ocorreu no período de agosto a novembro de 2019, estabelecidos os critérios de busca e seleção do material, tendo como critérios de inclusão: a) artigos publicados no recorte temporal de 2008 a 2019, jusstificado pelo ano de início da cirurgia robótica no Brasil; b) artigos nos idiomas português e inglês; $c$ ) artigos disponíveis na íntegra.

A pesquisa foi realizana na plataforma da Biblioteca Virtual de Saúde (BVS) e nas bases de dados da PubMed e Literatura Latino-Americana e do Caribe em Ciências da Saúde (LILACS), utilizado os descritores "robótica", "enfermagem" e "centro cirúrgico", associado com o operador booleano "AND/OR", tendo uma amostra inicial de 11 artigos publicados na LILACS e 89 publicados na PubMed. Artigos publicados em mais de uma base de dados foram contabilizados com um, com o intuíto de evitar duplicadas. 


\section{Fluxograma 1. Diagrama de fluxo de busca. Rio de Janeiro, RJ, Brasil, 2019}
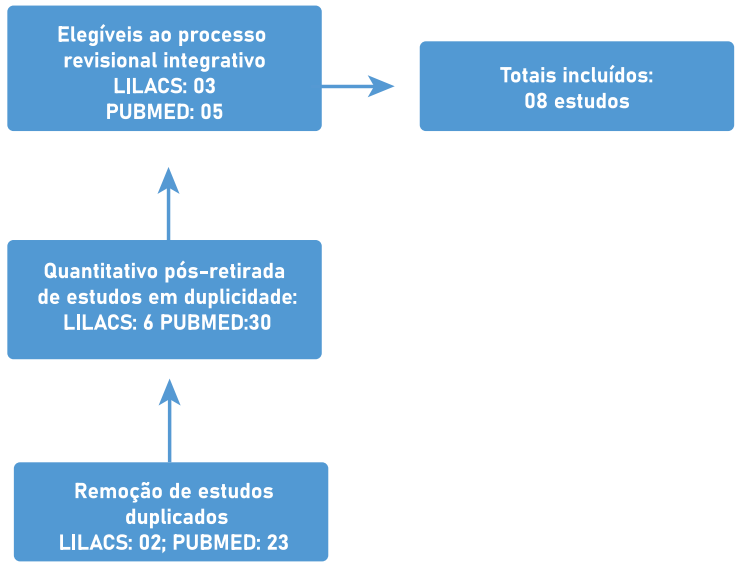

Após a aplicação dos critérios de inclusão e exclusão, leitura flutuante do título e do resumo do material, foram selecionados 08 artigos, conforme Fluxograma 1. Salientamos que a seleção do material foi realizada por dois pares de revisores.

Para seguir o rigor metodológico da revisão, foi elaborado pelos autores um instrumento próprio contendo as seguintes variáveis: número, periódico, autores, título, ano e síntese do artigo, com intuito viabilizar a organização, descrição e análise do material selecionado.

\section{RESULTADOS E DISCUSSÃO}

Foram selecionados 08 artigos para o estudo. Os anos de maiores publicações foram 2016, com $n=02$ estudos e 2017 também com $\mathrm{n}=02$ estudos dos estudos. A base de dados com maiores resultados foi a PubMed com $n=05$ estudos. Evidencia-se que as principais publicações sobre cirurgias robóticas concentram-se na área médica com enfoque na técnica cirúrgica, havendo uma carência de estudos com o foco na enfermagem e seu papel frente ao atendimento a esse paciente que é submetido à cirurgia com essa nova tecnologia.

\section{Quadro 1. Categorização dos artigos para análise de dados. Rio de Janeiro, RJ, Brasil, 2019}

\begin{tabular}{|c|c|c|c|c|c|}
\hline $\mathbf{N}$ & $\begin{array}{l}\text { PERIÓDICO } \\
\text { NOME DA REVISTA }\end{array}$ & AUTORES & TÍTULO & ANO & SIINTESE DO ARTIGO \\
\hline 1 & Rev. Bras. Enfermagem & Martins, et al. & $\begin{array}{l}\text { Atuação da } \\
\text { enfermagem em } \\
\text { cirurgias roboticas: } \\
\text { revisao integrativa. }\end{array}$ & 2019 & $\begin{array}{l}\text { Conhecer a produção cientifica sobre a atuacao } \\
\text { da equipe de enfermagem em cirurgias roboticas, } \\
\text { identificando-se papel do enfermeiro nos três } \\
\text { periodos do perioperatorio. }\end{array}$ \\
\hline 2 & Rev. SOBECC & Pinto, et al. & $\begin{array}{l}\text { Atuação do } \\
\text { enfermeiro na cirurgia } \\
\text { robótica: desafios e } \\
\text { perspectivas }\end{array}$ & 2018 & $\begin{array}{l}\text { Identificar os principais desafios e perspectivas } \\
\text { da atuação do enfermeiro na cirurgia robótica. }\end{array}$ \\
\hline 3 & $\begin{array}{l}\text { Editorial Office of Asian Journal } \\
\text { of Urology. }\end{array}$ & $\begin{array}{l}\text { Raheem, } \\
\text { et al. }\end{array}$ & $\begin{array}{l}\text { Robotic nurse duties in } \\
\text { the urology operative } \\
\text { room: } 11 \text { years of } \\
\text { experience }\end{array}$ & 2017 & $\begin{array}{c}\text { Relata sobre a cirurgia robótica urológica e } \\
\text { atuação da enfermagem }\end{array}$ \\
\hline 4 & Rev. Col. Bras. Cirurgia. & $\begin{array}{l}\text { Madureira, } \\
\text { et al }\end{array}$ & $\begin{array}{l}\text { Modelo de programa } \\
\text { de treinamento em } \\
\text { cirurgia robótica e } \\
\text { resultados iniciais }\end{array}$ & 2017 & $\begin{array}{l}\text { Descreve a implantação de um programa de } \\
\text { treinamento em cirurgia robóticas e sua utilização } \\
\text { em cirurgia geral. }\end{array}$ \\
\hline
\end{tabular}




\section{artigo}

Silva, F.F.; Santos, P.F.; Dalto, A.P.P.; Granandeiro, D.S.; Granandeiro, R.M.A.; Melo, N.G.S.; Passos, J.P.

Autonomia e gerenciamento do enfermeiro no serviço de cirurgia robótica

\begin{tabular}{|c|c|c|c|c|c|}
\hline 5 & $\begin{array}{c}\text { Administração Pública e } \\
\text { Gestão Social }\end{array}$ & Pitassi, et al. & $\begin{array}{l}\text { A cirurgia robótica nas } \\
\text { organizações públicas de } \\
\text { saúde: } 0 \text { caso do Instituto } \\
\text { Nacional de Câncer. }\end{array}$ & 2016 & $\begin{array}{c}\text { O estudo descreve como foi a implantação da } \\
\text { cirurgia robótica no primeiro hospital publico do } \\
\text { Brasil. }\end{array}$ \\
\hline 6 & Rev. SOBECC & $\begin{array}{l}\text { Souza, Bispo } \\
\text { e Cunha. }\end{array}$ & $\begin{array}{l}\text { Capacitação em cirurgia } \\
\text { robótica no programa de } \\
\text { residência em enfermagem } \\
\text { perioperatória }\end{array}$ & 2016 & $\begin{array}{l}\text { O estudo relata como se desenvolveu o } \\
\text { treinamento para atuação em cirurgia } \\
\text { robótica dentro do programa de residência de } \\
\text { enfermagem perioperatória. }\end{array}$ \\
\hline 7 & $\begin{array}{l}\text { Patient Safety in } \\
\text { Surgery }\end{array}$ & Song, et al. & $\begin{array}{l}\text { The second "time-out": a } \\
\text { surgical safety checklist }\end{array}$ & 2013 & $\begin{array}{l}\text { O artigo propõe a utilização de uma lista de } \\
\text { verificação de segurança em um segundo tempo } \\
\text { em cirurgias robóticas longas. }\end{array}$ \\
\hline 8 & $\begin{array}{l}\text { Instituto Nacional de } \\
\text { Telecomunicações } \\
\text { Inatel }\end{array}$ & $\begin{array}{l}\text { Rosa e } \\
\text { Moreira }\end{array}$ & $\begin{array}{l}\text { Aplicação da robótica nos } \\
\text { centros cirurgicos }\end{array}$ & 2013 & $\begin{array}{l}\text { Relata sobre a utilização de robótica no centro } \\
\text { cirúrgico e implantação do sistema Da Vinci no } \\
\text { Brasil. }\end{array}$ \\
\hline
\end{tabular}

A utilização da cirurgia robótica vem se ampliando rapidamente desde sua introdução, em 2001 em diversos campos cirúrgicos e em especialidades, como: a torácica, colorretal, hepatobiliar, ginecológica e urológica. Este avanço promove desafios em relação a custos, formação profissional e segurança do paciente ${ }^{(3)}$.

O emprego de tecnologias, como a laparoscópica, contribuiu para a ampliação da cirurgia minimamente invasiva avançando até a cirurgia robótica. Sendo assim, faz-se necessário que toda a equipe cirúrgica, inclusive os enfermeiros perioperatórios, se atualizem acerca de novas técnicas e adversidades que possam surgir envolvendo o uso destes novos recursos, com a finalidade de proporcionar segurança ao paciente ${ }^{(5)}$.

A robótica deu ao enfermeiro perioperatório a oportunidade de adaptar a sua prática, pensar criativamente e desenvolver práticas clínicas eficazes e seguras para o cuidado de seu pacient ${ }^{\mathrm{e}(6)}$. A atuação do enfermeiro em cirurgia robótica deve ser proativa e inclui diversas atribuições como fornecimento do instrumental, organização da equipe e segurança do paciente. A capacitação, tanto dos cirurgióes quanto para a equipe de enfermagem, deve ser rigorosa para que se possa implantar em um hospital o serviço de cirurgia robótica. Acrescenta-se que o papel do enfermeiro neste processo de aprendizado e evolução é essencial, pois é o profissional responsável por oferecer treinamento à toda equipe de enfermagem ${ }^{(7)}$.

A montagem da sala cirúrgica é uma das atribuições do enfermeiro. Revela-se que artigos destacam como imprescindível a organização da sala de operação de acordo com a cirurgia a ser realizada, onde o sistema robótico deve ser preparado adequadamente proporcionando condições técnicas para a execução da cirurgia. Faz- se necessário ofertar os instrumentais antes e durante o procedimento cirúrgico. No dia anterior à cirurgia e após a ocorrência de procedimento de outra especialidade, é preciso instalar o carrinho, console e sistema de visualização, conforme a cirurgia a ser efetuada ${ }^{(3)}$.

A segurança do paciente deve ser prioridade, para tal, o enfermeiro deve verificar os instrumentos, se certificando sobre seu perfeito funcionamento. Após a configuração do robô, o enfermeiro deve garantir que os instrumentos estejam em posição correta, além disso, deve estar familiarizado com os instrumentais robóticos utilizados de acordo com o tipo de procedimento a ser realizado ${ }^{(5)}$.

É importante ressaltar que o paciente deve ser fixado corretamente na mesa cirúrgica, utilizando-se faixas e coxins especiais com a finalida- de de evitar deslizes quando a mesa ciurgica é movimentada durante o procedimento $^{(7)}$.

Durante a cirurgia, o enfermeiro deve prezar pela segurança do paciente, verificar o sistema robótico, movimentos dos braços robóticos, nível de $\mathrm{CO} 2$ e se nenhuma parte do corpo do paciente esta sendo pressionada pela máquina, com a finalidade de evitar lesão(5).

A equipe de enfermagem deve estar preparada para interpretar e solucionar problemas exibidos pelo monitor do aparelho, isto é essencial para o sucesso do procedimento, pois permite que o cirurgião se concentre totalmente na execução da cirurgia. $\mathrm{O}$ enfermeiro também é responsável por anotar o nuúmero de vezes que um instrumental foi utilizado, de acordo com cada cirurgia, sendo assim, é sua tarefa estabelecer o quanto este material pode ser utilizado. Destaca-se que é importante que o enfermeiro possua noções básicas de software para que se sinta seguro e apto a atuar na cirurgia robótica ${ }^{(5)}$.

Destaca-se que é importante que o enfermeiro possua noções básicas de software para que se sinta seguro e apto a atuar na cirurgia robótica e, que há necessidade de mais programas educativos para enfermeiros que atuam na cirurgia robótica, que visem a atualização e garantam a oportuni- 
dade de aperfeiçoamento. Conseguimos identificar que grande parte dos treinamentos e educação continuada contempla apenas cirurgióes ${ }^{(5,6)}$.

A cirurgia robótica surge como um novo desafio para os profissionais de saúde, pois associa a técnica cirúrgica à tecnologia de ponta, exigindo que os profissionais da área renovem constantemente seus conhecimentos com o intuito de prestar um cuidado seguro ao paciente.

Neste contexto, o profissional enfermeiro figura como o principal responsável por garantir o melhor suporte possível, tanto à equipe quanto ao cliente. Para tanto, faz-se necessário que este profissional se empenhe em adquirir conhecimentos e atualizar-se frequentemente, pois além de gerenciar todo o processo, tem a responsabilidade de treinar a equipe de enfermagem de maneira efetiva para que saibam exatamente seu papel dentro da sala de cirurgia robótica, realizan- do seu trabalho com autoconfiança e eficiência ${ }^{(8)}$.

O trabalho gerencial do enfermeiro dentro da cirurgia robótica é multifacetado, pois deve verificar a marcação da cirurgia, prover o instrumental necessário, de acordo com o procedimento a ser realizado, deve preparar o robô e ser capaz de reconhecer problemas que ocorrem com o sistema, buscando soluções rapidamente. Além disso, sua responsabilidade é proporcionar a máxima segurança ao paciente, se atentando ao posicionamento correto, com a finalidade de evitar lesões ${ }^{(4)}$.

$O$ enfermeiro tem a incumbência de cuidar de toda a burocracia que envolve o procedimento cirúrgico. A sobrecarga burocrática que assola o enfermeiro, muita das vezes, prejudica o contato deste com o paciente e com o ato cirúrgico propriamente dito. Esse fato deve ser evitado, pois é preciso que o enfermeiro desenvolva a prática, uma vez que tem a função de educar sua equipe e fortalecer seu gerenciamento dentro da cirurgia robótica ${ }^{(9)}$.

\section{CONCLUSÃO}

O estudo possibilitou reconhecer a produção científica sobre a cirurgia robótica, desde sua origem e implantação, abordando suas caracteristicas gerais e identificando o papel da equipe cirurgica e de enfermagem. Evidenciou-se que o enfermeiro possui diversas atribuições, especialmente no que se refere à segurança do paciente, edu- cação continuada e gerenciamento de trodo o processo cirurgico, portanto necessita ser dinâmico e resolutivo em sua atuação. Constata-se que há escassez de material que destaque o gerenciamento do enfermeiro na cirurgia robótica, sendo assim, faz-se necessário o desenvolvimento de mais estudos nesta áera de atuação da enfermagem.

\section{REFERÊNCIAS}

1. SOBECC. Práticas Recomendadas SOBECC. 7 ed. rev. e atual. Associação Brasileira de Enfermeiros de Centro Cirúrgico, Recuperação Anestésica e Centro de Material e Esterilização. São Paulo, 201.

2. Madureira FAV, Varela JLS, Filho DM, D'Almeida LAV, Madureia FAV, Duarte AM, et al. Modelo de programa de treinamento em ciurgia robótica e resultados iniciais. Rev.Col. Bras.Cir [Internet]. 2017 [acesso em 20 out 2019]; 44(3):302307. Disponivel em: http://www.scielo.br/pdf/rcbc/v44n3/ 0100-6991-rcbc-44-03-0302.pdf.

3. Pinto EV, Lunardi LS, Treviso P. Botene DZA. Atuação do enfermeiro na cirurgia robótica: desafios e perspectivas. Rev. SOBECC [Internet]. 2018 [acesso em 11 nov 2019]; 23(1):4351. Disponivel em: https://revista.sobecc.org.br/sobecc/article/view/378.

4. Martins RC, Trevilato DD, Jost MT, Caregnato RCA. Nursing performance in robotic surgeries: integrative review. Rev Bras Enferm [Internet]. 2019 [acesso em 10 out 2019]; 72(3):795800. DOI: http://dx.doi.org/10.1590/0034-7167-2018-0426.

5. Raheem AA, Song HJ, Chang a KD, Choi a YD, Rha KH. Robotic nurse duties in the urology operative room: 11 years of experience. Asian Journal of Urology [Internet]. 2017 [acesso em 20 out 2019]; 4(2):116-123. Disponivel em: https://www.
ncbi.nlm.nih.gov/pmc/articles/PMC5717981/pdf/main.pdf.

6. Sousa CS, Bispo DM, Cunha ALM. Capacitação em cirurgia robótica no programa de residência em enfermagem perioperatória. Rev. SOBECC [Internet]. 2016 [acesso em 13 set 2019]; 21(4):198-202. Disponivel em: https://revista.sobecc. org.br/sobecc/article/view/27.

7. Ribeiro E, Ferraz KMC, Duran ECM. Atitude dos enfermeiros de centro cirúrgico diante da sistematização da assistência de enfermagem perioperatória. Rev. SOBECC [Internet]. 2017 [acesso em 13 set 2019]; 22(4):201-207. Disponivel em: https://revista.sobecc.org.br/sobecc/article/view/231.

8. Fonseca RMP, Peniche ACG. Enfermagem em centro cirúrgico: trinta anos após criação do sistema de assistência de enfermagem perioperatória. Acta Paul Enferm [Internet]. 2009 [acesso em 05 nov 2019]; 22(4):428-433. Disponível em: http://www.scielo.br/scielo.php?script=sci_abstract\&pid=S0103-21002009000400013\&lng=en\&nrm=iso\&tlng=pt.

9. Song JB, Goutham V, Mobley JM, Sam BB. The second "time-out": a surgical safety checklist for lengthy robotic surgeries. Patient Safety in Surgery [Internet]. 2013 [acesso em 10 nov 2019]; 7(1): 2-6. Disponivel em: https://www.ncbi.nlm.nih. gov/pubmed/23731776. 